\title{
Metabolic Effects of Chromium Supplementation in Dairy Cows in the Peripartal Period
}

\author{
A. PECHOVÁ, A. PODHORSKÝ, E. LOKAJOVÁ, L. PAVLATA, J. ILLEK \\ Clinic of Diseases of Ruminants, Faculty of Veterinary Medicine, \\ University of Veterinary and Pharmaceutical Sciences, Brno, Czech Republic
}

Received July 25, 2001

Accepted February 13, 2002

\begin{abstract}
Pechová A., A. Podhorský, E. Lokajová, L. Pavlata, J. Illek: Metabolic Effects of Chromium Supplementation in Dairy Cows in the Peripartal Period. Acta Vet. Brno 2002, 71: 9-18.

The aim of the work was to assess the effects of supplemental chromium $(\mathrm{Cr})$ on metabolism of dairy cows in the peripartal period. Rations fed to dairy cows in a herd of Holstein cattle with mean milk yield of 75001 were supplemented with chromium-enriched yeast (Co-Factor III Chromium Yeast, Alltech, $0.1 \% \mathrm{Cr}^{3+}$ ) at $10 \mathrm{mg}$ of $\mathrm{Cr}$ per animal per day. The treatment was started 21 days before the expected delivery date and discontinued 30 days after the delivery. Blood and urine samples were collected from ten experimental and ten control cows at weekly intervals, the state of health was monitored by regular clinical examinations, and milk yield for the first 100 days of lactation was recorded. The results indicate favourable effects of the supplementation on energy metabolism. The Cr-supplemented cows showed significantly higher blood glucose concentrations at post-partum (p.p.) weeks $4\left(4.25 \pm 0.21\right.$ vs. $\left.3.74 \pm 0.36 \mathrm{mmol}^{-1} \mathrm{l}^{-1} ; p<0.01\right)$ and $5\left(4.06 \pm 0.41 \mathrm{vs} .3 .640 .28 \mathrm{mmol} \cdot \mathrm{l}^{-1}\right.$; $p<0.05)$ and lower ketone bodies concentration at p.p. week $4\left(0.88 \pm 0.11 \mathrm{vs} .1 .38 \pm 0.66 \mathrm{mmol} \cdot \mathrm{l}^{-1}\right.$; $p$ < 0.05). The Cr-supplemented cows showed also significantly lower bilirubin concentration at p.p. week 2 (3.93 \pm 0.84 vs. $\left.6.47 \pm 3.25 \mu \mathrm{mol}^{-1} \mathrm{l}^{-1} ; p<0.05\right)$ and lower catalytic activities of aspartate aminotransferase at p.p. weeks $3\left(1.37 \pm 0.14\right.$ vs. $\left.1.66 \pm 0.20 \mu \mathrm{kat} \cdot \mathrm{l}^{-1} ; p<0.01\right)$ and $5(1.16 \pm 0.08 \mathrm{vs}$. $\left.1.47 \pm 0.18 \mathrm{ukat} \cdot \mathrm{l}^{-1} ; p<0.01\right)$ and lactate dehydrogenase at p.p. week $5(27.35 \pm 3.76 \mathrm{vs} .33 .61 \pm 5.61$ $\left.\mu \mathrm{kat} \cdot \mathrm{l}^{-1} ; p<0.05\right)$. No effects on the metabolism of nitrogen substances or minerals, insulin concentration in blood serum, and blood $\mathrm{Cr}$ concentration were observed. Chromium excretion in urine increased after parturition; higher concentrations were found in $\mathrm{Cr}$-supplemented cows at p.p. weeks 3 (7.14 \pm 1.72 vs. $\left.5.00 \pm 1.26 \mu \mathrm{g} \cdot \mathrm{l}^{-1} ; p<0.01\right)$ and $4\left(8.40 \pm 3.13\right.$ vs. $\left.4.04 \pm 1.32 \mu \mathrm{g} \cdot \mathrm{l}^{-1} ; p<0.01\right)$. Although chromium supplementation in the peripartal period significantly improved variables characterising the energy metabolism, no effects on milk yield for the first 100 days of lactation or on the incidence of clinical diseases were demonstrable.
\end{abstract}

Cattle, biochemical profile, liver, blood, chromium

The relevance of the trace element chromium $(\mathrm{Cr})$ to human and animal nutrition has been known for more than 40 years. $\mathrm{Cr}$ is present in the environment mostly in its trivalent form $\left(\mathrm{Cr}^{3+}\right)$, which is more stable than the hexavalent form $\left(\mathrm{Cr}^{6+}\right) . \mathrm{Cr}^{3+}$ is known for its in vivo antioxidative activity and favourable effects on the stability of proteins and nucleic acids (Anderson 1994). However, its most important metabolic effect consists in an enhancement of insulin activity by its presence in an organometallic molecule known as the glucose tolerance factor (GTF). Detailed structure of GTF is unknown, but it is assumed that the factor consists of $\mathrm{Cr}^{3+}$, nicotinic and glutamic acids, glycine, and cysteine (Ducros 1992). Most of Cr present in animal tissues is bound in GTF. Data on the distribution of chromium in animal tissues is rather sporadic. Low concentrations, declining with age (Mertz 1997), can be found in almost all tissues. In the man, the concentration is the highest in lung tissue and decreases in the following order: liver $\rightarrow$ pancreas $\rightarrow$ heart $\rightarrow$ striated muscles $\rightarrow$ kidney $\rightarrow$ spleen $\rightarrow$ brain (Lentner 1981). Blood concentration does not parallel that of tissue stores (Underwood 1977), but reflects exposure to chromium (Barceloux 1999). 
Cr enhances binding of insulin to cell membrane receptors and the optimisation of insulin activity results in better regulation of glucose uptake by cells, improved control of blood glucose concentration, and maximisation of the energetic potential. Consequences of $\mathrm{Cr}$ deficiency, which are probably associated with disturbed interaction between $\mathrm{Cr}$ and insulin, include lowering of glucose tolerance, increased insulin concentration, glycosuria, growth impairment, shortening of productive age, increased concentration of cholesterol and triacylglycerols, infertility, and peripheral neuropathies (Anders on 1994). Manifestations of Cr deficiency usually develop in animals affected by metabolic stress or exposed to physical strain.

Available data on $\mathrm{Cr}$ concentration in rations for farm animals is insufficient. Moreover, even rations containing $\mathrm{Cr}$ concentrations satisfying the need during a normal production period can become deficient in critical situations, such as advanced pregnancy, parturition, onset of lactation, weaning, transport, etc. The aim of the work was to assess the effects of peripartal supplemention of $\mathrm{Cr}$ on metabolism of dairy cows including possible ensuing effects on milk yield for the first 100 days of lactation and health.

Materials and Methods

The experiment was conducted in 1999 in South Moravia in a herd of Holstein cattle with 600 heads and a mean milk yield of 75001 (6900 1 in first-calvers and 79001 in $2^{\text {nd }}$-lactation and older cows). Twenty dairy cows, selected with regard to the expected delivery date and performance (analogous couple system), were divided into one experimental $(\mathrm{n}=10)$ and one control $(\mathrm{n}=10)$ groups, each including 5 first-calvers and five older cows. Chromiumenriched yeast (Co-Factor III Chromium Yeast, Alltech, $0.1 \% \mathrm{Cr}^{3+}$ ) was mixed into supplementary feed mixture at $10 \mathrm{mg}$ of $\mathrm{Cr}$ per animal per day mixed starting at expected delivery day -21 and discontinuing at post-delivery day 30 . Rations fed during the dry period consisted of corn silage $(17.0 \mathrm{~kg})$, whole-plant haylage $(4.0 \mathrm{~kg})$, minerals $(0.3$ $\mathrm{kg})$, and alfalfa hay $(3.0 \mathrm{~kg})$. Rations fed in first month of lactation were calculated for presumed milk yield 361 and consisted of corn silage $(20.0 \mathrm{~kg})$, whole-plant haylage $(8.0 \mathrm{~kg})$, alfalfa hay $(2.0 \mathrm{~kg})$, and concentrates $(12.5 \mathrm{~kg})$.

Blood and urine samples were collected regularly at weekly intervals starting 2 weeks before and discontinuing 5 weeks after delivery. The samples for plasma and whole blood analyses were collected from $v$. jugularis into disposable heparinised test tubes and analysed for concentrations of glucose, urea, triacylglycerols, calcium (Ca), magnesium (Mg), inorganic phosphorus (P) and catalytic activities of aspartate aminotransferase (AST), $\gamma$-glutamyl transferase (GMT), lactate dehydrogenase (LDH) in blood plasma, and of ketone bodies and chromium (Cr) in whole blood. The samples for blood serum analyses were collected into glass tubes and analysed for total bilirubin, cholesterol, albumin, nonesterified fatty acids (NEFA), cortisol, and insulin concentrations. Urine samples were collected using a plastic catheter and tested for $\mathrm{pH}$, density, concentrations of $\mathrm{Ca}, \mathrm{P}, \mathrm{Mg}$ and $\mathrm{Cr}$. Chromium concentrations in blood and urine were measured in post-partum weeks 1 to 4 only.

The following parameters were measured using the automatic analyser Cobas Mira (ROCHE, Switzerland) and the tests given in parentheses: urea (Urea UV KIN $4 \times 50$, Cat. No. 1307017), total bilirubin (BIL 100, Cat. No. 1105309), triacylglycerols (TGL $4 \times 100$, Cat. No. 1312983), GMT (GMT KIN 100, Cat. No. 1302082) the sets supplied by LACHEMA (Czech Republic); glucose ( ${ }^{\mathrm{L}}$ Glukosa, Cat. No. 11601), total protein (LProtein total, Cat. No. 12751), cholesterol ( ${ }^{\mathrm{L} C h o l e s t e r o l, ~ C a t . ~ N o .10851), ~ A S T ~(L A S T, ~ C a t . ~ N o . ~ 10351), ~ L D H ~(~}{ }^{\mathrm{L} L D H}$, Cat. No. 12352), P (LPosphorus inorganic, Cat. No. 11352) the sets supplied by BioVendor, (Czech Republic); albumin (Albumin liquicolor, Cat. No. 10560; set supplied by HUMAN, Germany); NEFA (NEFA, Cat, No. FA 115; set supplied by RANDOX, United Kingdom). The minerals $\mathrm{Ca}$ and $\mathrm{Mg}$ were determined by F-AAS using the spectrometer H 1550 (HILGER, Great Britain) apparatus. Cr was determined by ETA-AAS using the spectrometer SOLAAR 939 (UNICAM, Great Britain). Total ketone bodies (acetone, acetacetic acid, isopropanol, $\beta$-hydroxybutyric acid) were determined by gas chromatography (Chrom 5, LABORATORNÍ PŘÍSTROJE, Praha, Czech Republic), as described by Hradecký et al. (1978). Cortisol (LKCO1; set supplied by BioVendor, Czech Republic) and insulin (LKIN1; set supplied by BioVendor, Czech Republic) were determined by the chemiluminiscence technique using the apparatus Immulite (DPC, Los Angeles, USA).

The clinical state of the cows was monitored daily by the veterinarian in charge of the herd and data from performance checks were used for the assessment of milk yield for the first 100 days of lactation.

Variance of values obtained in the individual sets was analysed by the $F$-test and subsequently by the two-sided Student's $t$-test for sets with equal or unequal variance using the EXCEL software.

\section{Results}

Results of biochemical analyses are shown in Tables 1, 2 and 3. As the differences between the values of metabolic profile obtained in first-calvers and older cows were nonsignificant, the data obtained in the two subgroups were pooled. Energy deficit, 
Table 1

Selected values of energetic and protein metabolic profiles in control $(C ; n=10)$ and $\mathrm{Cr}$-supplemented $(\mathrm{E} ; \mathrm{n}=10)$ dairy cows ( $\mathrm{x}=$ mean; $\mathrm{s}=$ standard deviation)

\begin{tabular}{|c|c|c|c|c|c|c|c|c|c|}
\hline \multicolumn{2}{|c|}{$\begin{array}{c}\text { Weeks relative } \\
\text { to delivery }\end{array}$} & -2 & -1 & Delivery & 1 & 2 & 3 & 4 & 5 \\
\hline \multirow{2}{*}{$\begin{array}{l}\text { Glucose } \\
{\left[\mathrm{mmol} \cdot \mathrm{l}^{-1}\right]}\end{array}$} & $\begin{array}{ll}\mathbf{E} & \mathbf{x} \\
& \end{array}$ & $\begin{array}{l}3.90 \\
0.27\end{array}$ & $\begin{array}{l}3.71 \\
0.23\end{array}$ & $\begin{array}{l}3.70 \\
0.24\end{array}$ & $\begin{array}{l}4.23 \\
0.34\end{array}$ & $\begin{array}{l}4.24 \\
0.55\end{array}$ & $\begin{array}{l}4.15 \\
0.42\end{array}$ & $4.25 * *$ & $\begin{array}{l}4.06^{*} \\
0.40\end{array}$ \\
\hline & $\begin{array}{ll}\mathbf{C} & \mathbf{x} \\
& \mathbf{s}\end{array}$ & $\begin{array}{l}3.79 \\
0.23\end{array}$ & $\begin{array}{l}3.66 \\
0.28\end{array}$ & $\begin{array}{l}3.67 \\
0.22\end{array}$ & $\begin{array}{l}3.83 \\
0.60\end{array}$ & $\begin{array}{l}4.04 \\
0.59\end{array}$ & $\begin{array}{l}3.75 \\
0.48\end{array}$ & $\begin{array}{l}3.74 * * \\
0.36\end{array}$ & $\begin{array}{l}3.64 * \\
0.28\end{array}$ \\
\hline \multirow{4}{*}{$\begin{array}{c}\text { Total ketone } \\
\text { bodies } \\
{\left[\mathrm{mmol} \cdot \mathrm{l}^{-1}\right]}\end{array}$} & $\mathbf{E}$ & 0.88 & 0.96 & 0.88 & 0.93 & 1.13 & 0.95 & $0.88 *$ & 0.89 \\
\hline & $\mathbf{S}$ & 0.22 & 0.25 & 0.09 & 0.20 & 0.47 & 0.30 & 0.11 & 0.19 \\
\hline & $\mathbf{C} \quad \mathbf{x}$ & 0.76 & 0.80 & 0.84 & 1.29 & 1.29 & 1.46 & $1.38 \%$ & 1.10 \\
\hline & $\mathbf{S}$ & 0.09 & 0.30 & 0.14 & 0.75 & 0.46 & 1.06 & 0.66 & 0.24 \\
\hline \multirow{4}{*}{$\begin{array}{c}\text { Oxidized ketone } \\
\text { bodies } \\
{\left[\mu \mathrm{mol} \cdot \mathrm{I}^{-1}\right]}\end{array}$} & $\mathbf{E}$ & 22.1 & 30.8 & 29.8 & 36.0 & 100.5 & 68.2 & 31.4 & 33.7 \\
\hline & $\mathbf{S}$ & 4.6 & 13.8 & 3.5 & 13.3 & 13.3 & 9.2 & 10.5 & 25.1 \\
\hline & C $\quad x$ & 25.1 & 22.9 & 30.7 & 180.9 & 95.2 & 241.3 & 96.3 & 35.9 \\
\hline & $\mathbf{s}$ & 3.5 & 6.6 & 7.8 & 388.5 & 110.2 & 542.5 & 126.5 & 20.6 \\
\hline \multirow{4}{*}{$\begin{array}{c}\text { NEFA } \\
{\left[\mathrm{mmol}^{-1} \mathrm{l}^{-1}\right]}\end{array}$} & $\mathbf{E}$ & 0.22 & 0.15 & 0.20 & 0.26 & 0.28 & 0.22 & 0.21 & 0.13 \\
\hline & $\mathbf{s}$ & 0.15 & 0.07 & 0.10 & 0.12 & 0.21 & 0.12 & 0.15 & 0.09 \\
\hline & C $\quad \mathbf{x}$ & 0.12 & 0.25 & 0.18 & 0.24 & 0.33 & 0.24 & 0.19 & 0.35 \\
\hline & $\mathbf{S}$ & 0.06 & 0.15 & 0.10 & 0.08 & 0.15 & 0.15 & 0.10 & 0.27 \\
\hline \multirow{4}{*}{$\begin{array}{c}\text { Triacyl- } \\
\text { glycerols } \\
{\left[\mathrm{mmol} \cdot \mathrm{l}^{-1}\right]}\end{array}$} & $\mathbf{E}$ & 0.25 & 0.25 & 0.27 & 0.12 & 0.18 & 0.19 & $0.20 *$ & $0.17 * *$ \\
\hline & $\mathbf{S}$ & 0.05 & 0.04 & 0.05 & 0.04 & 0.07 & 0.05 & 0.06 & 0.04 \\
\hline & C $\quad x$ & 0.21 & 0.22 & 0.28 & 0.12 & 0.17 & 0.21 & $0.27 *$ & $0.27 * *$ \\
\hline & $\mathbf{S}$ & 0.05 & 0.08 & 0.07 & 0.05 & 0.09 & 0.06 & 0.07 & 0.04 \\
\hline \multirow{4}{*}{$\begin{array}{c}\text { Total } \\
\text { cholesterol } \\
{\left[\mathrm{mmol} \cdot \mathrm{l}^{-1}\right]}\end{array}$} & $\mathbf{E}$ & 3.10 & 3.40 & 3.36 & 3.21 & 3.79 & 4.21 & 4.47 & $4.02 *$ \\
\hline & $\mathbf{s}$ & 0.37 & 0.33 & 0.63 & 0.99 & 1.03 & 0.80 & 0.79 & 0.57 \\
\hline & $\begin{array}{ll}\mathbf{C} & \mathbf{x}\end{array}$ & 2.99 & 3.15 & 3.31 & 2.96 & 3.36 & 3.90 & 4.72 & $5.31 *$ \\
\hline & $\mathbf{s}$ & 0.53 & 0.54 & 0.77 & 0.91 & 0.90 & 1.03 & 1.35 & 1.18 \\
\hline \multirow{4}{*}{$\begin{array}{c}\text { Urea } \\
{\left[\mathrm{mmol} \cdot \mathrm{l}^{-1}\right]}\end{array}$} & & 3.78 & 2.52 & 2.20 & 4.00 & 3.65 & 4.35 & 3.93 & 4.33 \\
\hline & $\mathbf{S}$ & 1.06 & 0.53 & 0.72 & 1.53 & 1.18 & 0.80 & 1.04 & 1.61 \\
\hline & $\mathrm{C}$ & 4.45 & 2.37 & 2.14 & 4.12 & 3.84 & 4.58 & 4.65 & 4.22 \\
\hline & $\mathbf{S}$ & 1.03 & 1.41 & 0.64 & 1.22 & 1.20 & 1.05 & 0.89 & 0.81 \\
\hline \multirow{4}{*}{$\begin{array}{c}\text { Total protein } \\
{\left[\mathrm{g} \cdot \mathrm{l}^{-1}\right]}\end{array}$} & $\mathbf{E}$ & 73.24 & 66.76 & 67.31 & 68.05 & 77.37 & 78.68 & 77.47 & 80.82 \\
\hline & $\mathbf{S}$ & 3.54 & 7.53 & 5.48 & 5.73 & 3.20 & 5.38 & 6.83 & 5.43 \\
\hline & $\mathbf{C}$ & 73.06 & 69.77 & 66.12 & 67.04 & 73.68 & 80.55 & 78.98 & 78.36 \\
\hline & $\mathbf{S}$ & 2.66 & 3.05 & 1.9 & 3.31 & 3.91 & 3.53 & 5.05 & 4.35 \\
\hline \multirow{4}{*}{$\underset{\left[\mathrm{g} \cdot \mathrm{1}^{-1}\right]}{\operatorname{Albumin}}$} & $\mathbf{E}$ & 37.38 & 35.9 & 35.76 & 35.06 & 35.59 & 35.17 & 35.12 & 33.27 \\
\hline & $\mathbf{s}$ & 1.77 & 1.97 & 1.66 & 2.93 & 2.68 & 1.61 & 3.23 & 1.98 \\
\hline & C $\quad x$ & 37.46 & 37.16 & 36.45 & 36.40 & 34.91 & 35.44 & 35.82 & 36.00 \\
\hline & $\mathbf{S}$ & 1.10 & 1.47 & 1.68 & 2.03 & 2.59 & 2.67 & 3.34 & 2.40 \\
\hline
\end{tabular}

$* p<0.05$ (between groups $\mathrm{E}$ and $\mathrm{C}$ )

$* * p<0.01$ (between groups $\mathrm{E}$ and $\mathrm{C}$ )

manifested by low concentration of blood glucose under physiological range 3.0 $3.9 \mathrm{mmol} \cdot \mathrm{l}^{-1}$ (Vrzgula et al. 1990) and increased concentration of ketone bodies above physiological $0.1-1.0 \mathrm{mmol} \cdot \mathrm{l}^{-1}$ ( V rzgula et al. 1990), was found in many cows in the first month after delivery. This metabolic disturbance was more marked in the control group. The Cr-supplemented cows showed significantly higher blood glucose concentrations at postpartum (p.p.) weeks $4(p<0.01)$ and $5(p<0.05)$ and significantly lower concentration of total ketone bodies at p.p. week $5(p<0.05)$. Energy deficit was associated with damage to hepatic parenchyma manifested by increased activities of hepatic enzymes and higher concentration of total bilirubin in blood serum (Table 2). This damage was less serious in the experimental group which showed significantly lower total bilirubin concentration at p.p. week $2(p<0.05)$ and lower catalytic activities of AST at p.p. weeks 3 and $5(p<0.01)$ and LDH at p.p. week $5(p<0.05)$. Marked increase in the catalytic activity of AST above physiological range $0.72-1.41 \mu \mathrm{kat} \cdot \mathrm{l}^{-1}$ (Pechová et al. 1997) after delivery was demonstrated in $40 \%$ cows of the Cr-supplemented and $90 \%$ of the control cows. This 
Table 2

Selected values of metabolic profiles in control $(C ; n=10)$ and $C r$-supplemented $(E ; n=10)$ dairy cows $(\mathrm{x}=$ mean; $\mathrm{s}=$ standard deviation $)$

\begin{tabular}{|c|c|c|c|c|c|c|c|c|c|c|}
\hline \multicolumn{3}{|c|}{$\begin{array}{l}\text { Weeks relative } \\
\text { to delivery }\end{array}$} & -2 & -1 & Delivery & 1 & 2 & 3 & 4 & 5 \\
\hline \multirow{4}{*}{$\begin{array}{c}\text { Total } \\
\text { bilirubin } \\
{\left[\mu \mathrm{mol} \cdot 1^{-1}\right]}\end{array}$} & $\mathbf{E}$ & $\mathbf{x}$ & 2.74 & 3.51 & 3.09 & 8.45 & $3.93 *$ & 3.67 & 3.50 & 2.47 \\
\hline & & $\mathbf{S}$ & 1.08 & 1.25 & 0.89 & 5.52 & 0.84 & 1.45 & 1.62 & 0.86 \\
\hline & C & $\mathbf{x}$ & 2.90 & 3.77 & 4.69 & 7.39 & $6.47 *$ & 6.39 & 4.79 & 3.38 \\
\hline & & $\mathbf{s}$ & 0.67 & 1.21 & 2.79 & 3.51 & 3.25 & 3.56 & 2.08 & 1.28 \\
\hline \multirow{4}{*}{$\underset{\left[\mu \mathrm{kat} \cdot 1^{-1}\right]}{\mathbf{A S T}}$} & $\mathbf{E}$ & $\mathbf{x}$ & 1.12 & 1.13 & 1.14 & 1.69 & 1.69 & $1.37 * *$ & 1.34 & $1.16^{* * *}$ \\
\hline & & $\mathbf{s}$ & 0.16 & 0.16 & 0.20 & 0.58 & 0.47 & 0.14 & 0.27 & 0.08 \\
\hline & C & $\mathbf{x}$ & 1.09 & 1.10 & 1.16 & 1.73 & 1.84 & $1.66 * *$ & 1.58 & $1.47 * *$ \\
\hline & & $\mathbf{s}$ & 0.15 & 0.12 & 0.14 & 0.32 & 0.56 & 0.20 & 0.25 & 0.18 \\
\hline \multirow{4}{*}{$\underset{\left[\mu \mathrm{kat} \cdot \cdot^{-1}\right]}{\mathbf{L D H}}$} & $\mathbf{E}$ & $\mathbf{x}$ & 27.65 & 25.78 & 24.96 & 30.75 & 29.49 & 28.37 & 31.89 & $27.35^{*}$ \\
\hline & & $\mathbf{S}$ & 3.86 & 2.46 & 3.18 & 7.42 & 5.11 & 5.92 & 6.52 & 3.76 \\
\hline & C & $\mathbf{x}$ & 29.69 & 28.77 & 28.09 & 33.76 & 35.82 & 34.37 & 36.63 & $33.61 *$ \\
\hline & & $\mathbf{s}$ & 2.37 & 3.37 & 3.58 & 8.50 & 7.83 & 6.49 & 8.27 & 5.61 \\
\hline \multirow{4}{*}{$\begin{array}{c}\text { GMT } \\
{\left[\mu \mathrm{kat} \cdot \mathrm{I}^{-1}\right]}\end{array}$} & $\mathbf{E}$ & $\mathbf{x}$ & 0.26 & 0.29 & 0.35 & 0.42 & 0.42 & 0.37 & 0.39 & 0.40 \\
\hline & & $\mathbf{s}$ & 0.04 & 0.06 & 0.08 & 0.10 & 0.09 & 0.09 & 0.07 & 0.07 \\
\hline & $\mathbf{C}$ & $\mathbf{x}$ & 0.29 & 0.32 & 0.38 & 0.47 & 0.48 & 0.45 & 0.46 & 0.47 \\
\hline & & $\mathbf{s}$ & 0.05 & 0.09 & 0.11 & 0.19 & 0.19 & 0.13 & 0.19 & 0.18 \\
\hline \multirow{4}{*}{$\begin{array}{l}\text { Cortisol } \\
{\left[\mathrm{nmol} \cdot \mathrm{l}^{-1}\right]}\end{array}$} & $\mathbf{E}$ & $\mathbf{x}$ & $53.22 *$ & 41.14 & 39.67 & 61.56 & 32.41 & 33.29 & 53.29 & 42.15 \\
\hline & & $\mathbf{s}$ & 25.46 & 25.48 & 15.43 & 57.40 & 16.77 & 25.73 & 33.40 & 25.28 \\
\hline & $\mathbf{C}$ & $\mathbf{x}$ & $25.84 *$ & 30.23 & 33.88 & 35.78 & 36.15 & 34.78 & 35.99 & 36.13 \\
\hline & & $\mathbf{s}$ & 6.10 & 6.01 & 15.20 & 19.61 & 20.75 & 23.43 & 26.00 & 15.14 \\
\hline \multirow{4}{*}{$\begin{array}{l}\text { Insulin } \\
{\left[\mathrm{nmol} \cdot \mathrm{I}^{-1}\right]}\end{array}$} & $\mathbf{E}$ & $\mathbf{x}$ & 4.93 & 5.49 & 6.64 & 5.80 & 3.77 & 2.39 & 3.78 & 3.57 \\
\hline & & $\mathbf{s}$ & 4.20 & 3.10 & 4.35 & 3.00 & 2.69 & 1.01 & 2.45 & 1.60 \\
\hline & $\mathbf{C}$ & $\mathbf{x}$ & 3.80 & 5.31 & 5.48 & 4.58 & 3.84 & 2.18 & 3.25 & 2.52 \\
\hline & & $\mathbf{s}$ & 0.87 & 4.65 & 2.85 & 3.37 & 2.34 & 0.37 & 1.27 & 0.75 \\
\hline
\end{tabular}

$* p<0.05$ (between groups $\mathrm{E}$ and $\mathrm{C}$ )

$* * p<0.01$ (between groups $\mathrm{E}$ and $\mathrm{C}$ )

increase persisted in 8 of the control cows, but in only $3 \mathrm{Cr}$-supplemented cows throughout the post-partum period. More serious damage to hepatic function manifested by increased catalytic activity of GMT above physiological range $0.14-0.55 \mu \mathrm{kat} \cdot \mathrm{l}^{-1}$ (Pechová et al. 1997) persisting for 2 to 6 weeks was found in 4 control cows. A similar, but transient increase, demonstrated on one sampling only, was observed in $2 \mathrm{Cr}$-supplemented cows. The between-group difference was nonsignificant, however. The concentration of total cholesterol in blood plasma increased progressively after delivery throughout the rest of the observation period and reached significantly higher values in the control than in the $\mathrm{Cr}$ supplemented cows $(p<0.05)$ at p.p. week 5. The concentration of triacylglycerols in blood moderately decreased in the post-partum period and although the concentrations found at p.p. weeks 4 and 5 were significantly ( $p<0.05$ and $p<0.01$, respectively) lower in the $\mathrm{Cr}$ treated than in the control cows.

Supplemental $\mathrm{Cr}$ did not influence the metabolism of nitrogen substances (Table 1). The dynamics of blood urea concentration reflected the composition of rations and were similar in both the groups. Total protein and albumin concentrations were uniform throughout the observation period and did not exceed the physiological range 60-80 g. $\mathrm{l}^{-1}, 30-42 \mathrm{~g} \cdot \mathrm{l}^{-1}$, respectively (Vrzgula et al. 1990).

The concentration of cortisol in blood plasma of cows was monitored as an index of stress severity. However, their interpretation is rather problematic owing to large individual variations (variance coefficient $20-93 \%$ ). The concentration rose above the normal level of 15 to $19 \mathrm{nmol}^{-1}$ (Kaneko 1997) in $70 \%$ of the Cr-treated and the control cows. Increase above $100 \mathrm{nmol} \cdot \mathrm{l}^{-1}$ recorded in two of the experimental cows was responsible for a high variance coefficient (93\%). The concentrations tended to be higher in the Cr-treated cows, but a significant difference $(p<0.05)$ was found only two weeks before delivery. The 
concentration of insulin was monitored because the effects of $\mathrm{Cr}$ are closely associated with the activity of this hormone. The concentration of insulin decreased in the $1^{\text {st }}$ and $2^{\text {nd }}$ weeks after delivery and lower levels persisted up to the end of the observation period. No significant between-group differences were found.

Mineral metabolism (Table 3) was assessed on the basis of urine and blood plasma analyses. No significant between-group differences indicative of effects of supplemental $\mathrm{Cr}$ on the metabolism of $\mathrm{Ca}, \mathrm{P}$ and $\mathrm{Mg}$ were demonstrable.

Table 3

Selected values of mineral profiles in control $(C ; n=10)$ and $C r$-supplemented $(E ; n=10)$ dairy cows $(\mathrm{x}=$ mean $; \mathrm{s}=$ standard deviation $)$

\begin{tabular}{|c|c|c|c|c|c|c|c|c|c|c|}
\hline \multicolumn{3}{|c|}{$\begin{array}{c}\text { Weeks relative } \\
\text { to delivery }\end{array}$} & -2 & -1 & Delivery & 1 & 2 & 3 & 4 & 5 \\
\hline \multirow{4}{*}{$\begin{array}{c}\text { Ca-plasma } \\
{\left[\mathrm{mmol} \cdot \mathrm{I}^{-1}\right]}\end{array}$} & $\mathbf{E}$ & $\mathbf{x}$ & 2.46 & 2.52 & 2.49 & 2.32 & 2.55 & 2.45 & 2.39 & 2.33 \\
\hline & & $\mathbf{S}$ & 0.22 & 0.13 & 0.18 & 0.37 & 0.22 & 0.12 & 0.23 & 0.11 \\
\hline & $\mathbf{C}$ & $\mathbf{x}$ & 2.43 & 2.55 & 2.53 & 2.33 & 2.36 & 2.43 & 2.42 & 2.52 \\
\hline & & $\mathbf{s}$ & 0.09 & 0.11 & 0.13 & 0.11 & 0.15 & 0.18 & 0.19 & 0.14 \\
\hline \multirow{4}{*}{$\begin{array}{l}\text { P-plasma } \\
{\left[\mathrm{mmol}^{-1} \mathrm{I}^{-1}\right]}\end{array}$} & $\mathbf{E}$ & $\mathbf{x}$ & 2.40 & 2.45 & 2.18 & 1.66 & 1.92 & 1.88 & 1.89 & 1.90 \\
\hline & & $\mathbf{S}$ & 0.30 & 0.41 & 0.32 & 0.42 & 0.27 & 0.44 & 0.49 & 0.12 \\
\hline & C & $\mathbf{x}$ & 2.16 & 2.20 & 1.91 & 1.52 & 1.76 & 1.81 & 1.91 & 1.72 \\
\hline & & $\mathbf{s}$ & 0.30 & 0.26 & 0.30 & 0.26 & 0.31 & 0.29 & 0.30 & 0.25 \\
\hline \multirow{4}{*}{$\underset{\left[\mathrm{mmol} \cdot \cdot^{-1}\right]}{\text { Mg-plasma }}$} & $\mathbf{E}$ & $\mathbf{x}$ & 0.82 & 0.79 & 0.84 & 0.98 & 0.83 & 0.85 & 0.91 & 0.93 \\
\hline & & $\mathbf{s}$ & 0.09 & 0.08 & 0.07 & 0.20 & 0.10 & 0.09 & 0.07 & 0.08 \\
\hline & $\mathbf{C}$ & $\mathbf{X}$ & 0.82 & 0.81 & 0.83 & 0.83 & 0.80 & 0.82 & 0.85 & 0.99 \\
\hline & & $\mathbf{s}$ & 0.03 & 0.08 & 0.08 & 0.09 & 0.13 & 0.12 & 0.17 & 0.15 \\
\hline \multirow{4}{*}{$\begin{array}{l}\text { Ca-urine } \\
{\left[\mathrm{mmol} \cdot \mathrm{I}^{-1}\right]}\end{array}$} & $\mathbf{E}$ & $\mathbf{x}$ & 0.91 & 0.22 & 0.61 & 0.16 & 0.36 & 0.17 & 0.28 & 0.13 \\
\hline & & $\mathbf{S}$ & 1.22 & 0.15 & 0.88 & 0.06 & 0.33 & 0.10 & 0.33 & 0.03 \\
\hline & $\bar{C}$ & $\mathbf{x}$ & 0.19 & 0.51 & 0.59 & 0.35 & 0.28 & 0.72 & 0.95 & 0.28 \\
\hline & & $\mathbf{s}$ & 0.18 & 0.50 & 0.79 & 0.46 & 0.42 & 1.16 & 2.45 & 0.14 \\
\hline \multirow{4}{*}{$\begin{array}{c}\text { P-urine } \\
{\left[\mathrm{mmol}^{-1} \mathrm{l}^{-1}\right]}\end{array}$} & $\mathbf{E}$ & $\mathbf{x}$ & 2.24 & 1.88 & 2.46 & 2.54 & 2.73 & 1.46 & 2.07 & 3.25 \\
\hline & & $\mathbf{s}$ & 1.37 & 1.49 & 3.79 & 2.43 & 3.50 & 1.73 & 2.02 & 3.74 \\
\hline & $\mathbf{C}$ & $\mathbf{x}$ & 4.11 & 2.41 & 3.98 & 1.97 & 1.85 & 2.24 & 1.69 & 1.25 \\
\hline & & $\mathbf{s}$ & 2.93 & 1.79 & 5.41 & 3.01 & 1.54 & 2.81 & 1.43 & 1.54 \\
\hline \multirow{4}{*}{$\begin{array}{l}\text { Mg-urine } \\
{\left[\mathrm{mmol} \cdot \mathrm{l}^{-1}\right]}\end{array}$} & $\mathbf{E}$ & $\mathbf{x}$ & 12.72 & 11.65 & 13.66 & 7.51 & 6.22 & 6.80 & 9.06 & 9.37 \\
\hline & & $\mathbf{s}$ & 6.23 & 4.11 & 4.27 & 5.24 & 4.50 & 2.62 & 4.09 & 3.48 \\
\hline & $\mathbf{C}$ & $\mathbf{X}$ & 13.19 & 10.52 & 9.56 & 5.94 & 7.49 & 7.50 & 6.86 & 10.20 \\
\hline & & $\mathbf{s}$ & 4.19 & 3.30 & 4.05 & 3.52 & 3.67 & 5.03 & 3.04 & 3.34 \\
\hline
\end{tabular}

Chromium concentrations in urine and blood samples are given in Table 4. The supplementation had no significant effect on whole blood concentration of $\mathrm{Cr}$ and no marked fluctuations were observed during the observation period. Increased urinary excretion of $\mathrm{Cr}$ was recorded in the $\mathrm{Cr}$-treated cows throughout the postparturient period; significant between-group differences were found at weeks 3 and 4 ( $p<0.01)$.

Clinical examination did not reveal significant health disorders in any of the cows. One of the Cr-supplemented cows developed puerperal paresis, but responded promptly to infusion therapy. Retention of placenta with ensuing endometritis was diagnosed in $2 \mathrm{Cr}-$ supplemented and 3 control cows. One of the two affected experimental cows gave birth to twins in a complicated delivery.

Performance was assessed in terms of milk yield and composition for the first 100 days of lactation (Table 5). No significant difference in any of the parameters was found between the Cr-supplemented and the control groups.

\section{Discussion}

Investigation of health and metabolic effects of supplemental $\mathrm{Cr}$ in dairy cows, focused on the terminal stage of pregnancy and early lactation as a period when the animals are exposed to increased metabolic stress and physical strain, demonstrated favourable effects 
Table 4

Blood and urinary concentrations of $\mathrm{Cr}$ in control $(\mathrm{C} ; \mathrm{n}=10)$ and $\mathrm{Cr}$-supplemented $(\mathrm{E} ; \mathrm{n}=10)$ dairy cows $(\mathrm{x}=$ mean $; \mathrm{s}=$ standard deviation $)$

\begin{tabular}{|c|c|c|c|c|c|c|c|}
\hline \multicolumn{3}{|c|}{$\begin{array}{l}\text { Weeks relative } \\
\text { to delivery }\end{array}$} & Delivery & 1 & 2 & 3 & 4 \\
\hline \multirow{2}{*}{$\begin{array}{l}\text { Blood } \\
{\left[\mu \mathrm{g} \cdot 1^{-1}\right]}\end{array}$} & $\mathbf{E}$ & $\begin{array}{l}\mathbf{X} \\
\mathbf{S}\end{array}$ & $\begin{array}{l}4.20 \\
2.32\end{array}$ & $\begin{array}{l}3.09 \\
1.20\end{array}$ & $\begin{array}{l}3.65 \\
2.64\end{array}$ & $\begin{array}{l}4.55 \\
2.62\end{array}$ & $\begin{array}{l}4.86 \\
2.68\end{array}$ \\
\hline & $\mathbf{C}$ & $\begin{array}{l}\mathbf{x} \\
\mathbf{S}\end{array}$ & $\begin{array}{l}3.37 \\
2.31 \\
\end{array}$ & $\begin{array}{l}4.38 \\
1.53 \\
\end{array}$ & $\begin{array}{l}3.77 \\
1.72 \\
\end{array}$ & $\begin{array}{l}3.81 \\
0.86 \\
\end{array}$ & $\begin{array}{l}4.18 \\
2.17 \\
\end{array}$ \\
\hline \multirow{2}{*}{$\begin{array}{c}\text { Urine } \\
{\left[\mu \mathrm{g} \cdot 1^{-1}\right]}\end{array}$} & $\mathbf{E}$ & $\begin{array}{l}\mathbf{X} \\
\mathbf{S} \\
\end{array}$ & $\begin{array}{l}6.08 \\
2.39 \\
\end{array}$ & $\begin{array}{l}9.11 \\
4.03 \\
\end{array}$ & $\begin{array}{l}7.08 \\
3.30 \\
\end{array}$ & $\begin{array}{l}7.14 * * \\
1.72\end{array}$ & $\begin{array}{l}8.40 * * \\
3.13\end{array}$ \\
\hline & $\mathbf{C}$ & $\begin{array}{l}\mathbf{X} \\
\mathbf{S}\end{array}$ & $\begin{array}{l}6.36 \\
4.43 \\
\end{array}$ & $\begin{array}{l}7.65 \\
2.61 \\
\end{array}$ & $\begin{array}{l}6.46 \\
1.82 \\
\end{array}$ & $\begin{array}{l}5.00 * * \\
1.26\end{array}$ & $\begin{array}{l}4.04 * * \\
1.32\end{array}$ \\
\hline
\end{tabular}

** $p<0.01$ (between groups $\mathrm{E}$ and $\mathrm{C}$ )

Table 5

Performance of dairy cows for the first 100 days of lactation in the control (C) and the experimental (E) groups ( $\mathrm{x}=$ mean; $\mathrm{s}=$ standard deviation, $\mathrm{n}=$ number of cows)

\begin{tabular}{|c|c|c|c|c|c|c|c|}
\hline & \multicolumn{3}{|c|}{$n$} & Milk yield (1) & Fat $(\%)$ & Protein $(\%)$ & Lactose $(\%)$ \\
\hline \multirow{2}{*}{$\begin{array}{c}\text { First- } \\
\text { calvers }\end{array}$} & $\mathbf{E}$ & 5 & $\begin{array}{l}\mathbf{x} \\
\text { S }\end{array}$ & $\begin{array}{r}2277.3 \\
412.3\end{array}$ & $\begin{array}{l}3.80 \\
0.49\end{array}$ & $\begin{array}{l}3.49 \\
0.25\end{array}$ & $\begin{array}{l}5.02 \\
0.06\end{array}$ \\
\hline & $\mathbf{C}$ & 5 & $\begin{array}{l}\mathbf{x} \\
\mathbf{S}\end{array}$ & $\begin{array}{r}2675.4 \\
265.0\end{array}$ & $\begin{array}{l}3.91 \\
0.40\end{array}$ & $\begin{array}{l}3.48 \\
0.12\end{array}$ & $\begin{array}{l}0.04 \\
0.11\end{array}$ \\
\hline \multirow{2}{*}{ Cows } & $\mathbf{E}$ & 5 & $\begin{array}{l}\mathbf{x} \\
\mathrm{S}\end{array}$ & $\begin{array}{r}2966.6 \\
160.2\end{array}$ & $\begin{array}{l}4.04 \\
0.43\end{array}$ & $\begin{array}{l}3.34 \\
0.20\end{array}$ & $\begin{array}{l}5.08 \\
0.18\end{array}$ \\
\hline & $\mathbf{C}$ & 5 & $\begin{array}{l}\mathbf{x} \\
\mathbf{s}\end{array}$ & $\begin{array}{r}3068.0 \\
404.8\end{array}$ & $\begin{array}{l}4.26 \\
0.58\end{array}$ & $\begin{array}{l}3.23 \\
0.23\end{array}$ & $\begin{array}{l}4.95 \\
0.19\end{array}$ \\
\hline \multirow{2}{*}{ Total } & $\mathbf{E}$ & 10 & $\begin{array}{l}\mathbf{x} \\
\mathbf{s}\end{array}$ & $\begin{array}{r}2594.2 \\
472.8 \\
\end{array}$ & $\begin{array}{l}3.94 \\
0.47\end{array}$ & $\begin{array}{l}3.41 \\
0.23\end{array}$ & $\begin{array}{l}5.06 \\
0.14\end{array}$ \\
\hline & $\mathbf{C}$ & 10 & $\begin{array}{l}\mathbf{x} \\
\mathbf{S}\end{array}$ & $\begin{array}{r}2871.7 \\
394.4\end{array}$ & $\begin{array}{l}4.09 \\
0.53\end{array}$ & $\begin{array}{l}3.36 \\
0.22\end{array}$ & $\begin{array}{l}5.00 \\
0.16\end{array}$ \\
\hline
\end{tabular}

of this treatment on energy metabolism in the puerperal period. The latter effect was apparently associated with increased gluconeogenesis in Cr-supplemented cows ( $\mathrm{S}$ ano et al. 1997; Subiyatno et al. 1996). Such result is indicative of a less severe and shorter lasting energy deficit. Chang et al. (1996) and Besong et al. (1996) reported a lower incidence of ketosis in multiparous cows. On the other hand, Yang et al. (1996) and Ireland (1999) did not demonstrate any decrease in NEFA and $\beta$-hydroxybutyrate concentrations in the blood of dairy cows. An increase in gluconeogenesis and glycogenolysis in Cr-supplemented cows was presumed also by Subiyatno et al. (1996) who demonstrated that such treatment tended to reduce the insulin:glucagon ratio. Less severe damage to the hepatic parenchyma in the postpartum period was apparently associated with a lower degree of energy deficit. We assume that this effect does not result from direct favourable action of $\mathrm{Cr}$ on the hepatic parenchyma, but possible toxicity of $\mathrm{Cr}$ at the dose used in our experiment cannot be disregarded. Similarly, B e s ong et al. (1996) found a decrease in hepatic triacylglycerol concentration in cows receiving $\mathrm{Cr}$ supplements.

Certain between-group differences were found in total blood serum cholesterol concentration. Studies of effects of Cr supplementation on the metabolism of cholesterol in humans demonstrated an increase in the concentration of HDL cholesterol and decreases in concentrations of triacylglycerols and total and LDL cholesterol (Anderson 1995). Studies of cholesterol concentration in dairy cows are encouraged by the fact that cholesterol influences the production of steroid hormones; hence, low cholesterol concentration can result in a disturbance of reproductive functions (Williams 1994). We therefore assume 
that the metabolism of cholesterol in cows should be considered in studies of effects of supplemental $\mathrm{Cr}$.

Blood serum concentrations of cortisol found in our experiment showed wide variations and it is therefore difficult to assess the effects of $\mathrm{Cr}$ thereon. Individual variability in responses of cows to stress factors apparently played a more important role than supplemental $\mathrm{Cr}$. Mean cortisol concentrations were higher in the $\mathrm{Cr}$-supplemented than in the control cows. Chang and Mowat (1992) and Mow at et al. (1993) demonstrated a decrease in cortisol concentration by 19 to $27 \%$ in calves exposed to transport stress. In another study, Moonsie-Shager and Mowat (1993) demonstrated in calves an inverse linear proportion between dosage of $\mathrm{Cr}$ and blood serum cortisol concentration. Although this finding has been confirmed in repeated studies, the situation in cows in the peripartal period is not thus unequivocal. Subiy at no et al. (1993) demonstrated a decrease of cortisol concentrations after calving in Cr-supplemented cows. On the other hand, our results confirm the data of Yang et al. (1996) and Burton et al. (1995) who reported an inconsistent increase in cortisol concentrations in animals receiving $\mathrm{Cr}$ supplements. It is evident that the $\mathrm{Cr}$ - cortisol relation is not as unequivocal as believed earlier.

A moderate decrease in insulin concentration after delivery was observed in all the cows irrespective of $\mathrm{Cr}$ supplementation. Similar findings were published also by B e s ong et al. (1996) and Ireland (1999). However, a certain relation between $\mathrm{Cr}$ and insulin concentration is apparent from the results published by Frank et al. (2000) who demonstrated increased insulin concentrations in Cr-deficient goats. The lower rate of lipomobilisation in the cows receiving $\mathrm{Cr}$ supplements may consist in the effect of $\mathrm{Cr}$ on the biological response to insulin. Such interpretation is supported by the finding of equal biological responses in Cr-supplemented cows showing lower insulin concentration (Mallard and Borg s 1997). However, other authors assume a higher insulin resistance in energy-deficient first-calvers in early lactation and thereby explain favourable effects of $\mathrm{Cr}$ supplementation on milk yield in first-calvers (Mow at et al. 1995).

Papers presenting data on $\mathrm{Cr}$ concentrations in tissues and body fluids are rather scarce. Our investigations included determination of blood and urinary $\mathrm{Cr}$ concentrations. Chromium is known to be present in blood as free $\mathrm{Cr}^{3+}$ or bound to transferrin, other proteins, or complexes, such as the glucose tolerance factor GTF-Cr (Ducros 1992). Cr supplementation had no effect on its blood concentration. No significant differences were found between the $\mathrm{Cr}$-supplemented and the control groups. The relationship between blood $\mathrm{Cr}$ concentration and the content of $\mathrm{Cr}$ in grazed plants in an area with an increased $\mathrm{Cr}$ burden was studied by S a hin et al. (1996). Blood $\mathrm{Cr}$ concentrations ranged from 9 to $92 \mu \mathrm{g} \cdot \mathrm{l}^{-1}$ and were dependent on the content of $\mathrm{Cr}$ in plants. Although blood $\mathrm{Cr}$ concentrations reflect to a certain extent the intake of this element, their values can be relevant only in cases of excessive $\mathrm{Cr}$ intake. This is evident from our studies in which supplementation with $10 \mathrm{mg}$ of $\mathrm{Cr}$ had no effect on blood $\mathrm{Cr}$ concentration. Blood $\mathrm{Cr}$ concentration cannot be used as an indicator of Cr status, because it is not balanced with tissue Cr stores (Underwood 1977). Absorbed $\mathrm{Cr}$ is excreted above all in urine and the excreted amount is controlled by several factors. Our results indicate a certain increase in urinary $\mathrm{Cr}$ excretion in the puerperal period which was more marked in the Cr-supplemented than in the control group. The increase apparently resulted from stress and a higher proportion of saccharides in the ration as described by Mordenti et al. (1997). The difference between the Cr-supplemented and the control cows is consistent with results of other authors who tested the use of urinary excretion of $\mathrm{Cr}$ as a biological marker in humans exposed to toxic doses of $\mathrm{Cr}$ (Paustenbach et al. 1997). Considering rather high standard deviations and the small difference between the treated and the control groups, monitoring of urinary excretion of $\mathrm{Cr}$ in cattle is apparently of limited value only. 
No unequivocal effect of $\mathrm{Cr}$ supplementation on the state of health was demonstrable in our study. Although the prevalence of retained placenta and endometritis was somewhat higher in the control group, the number of cases is too low for drawing any conclusions. Similar results were reported also by Chang et al. (1996) who did not observe any effects of Cr supplementation on the prevalence of mastitis, oedema, or abomasal displacement and found only a somewhat lower prevalence of retained placenta and endometritis in the treated cows. On the other hand, Villalobos et al. (1997), who started Cr supplementation nine weeks before the expected delivery date, reported a decrease in the prevalence of retained placenta to almost one fourth of the value observed in untreated cows.

No effect of $\mathrm{Cr}$ supplementation of dairy cows in peripartal period on performance for the first 100 day of lactation was demonstrable in our experiment. Data published by other authors are conflicting; some reported an increase in milk yield (Hayirli et al. 2001), other found such an effect only in first-calvers (Y ang et al. 1996), and some were unable to demonstrate any favourable effect of $\mathrm{Cr}$ supplementation on milk yield (Subi y a tno et al. 1996). Unlike the above authors, we did not investigate direct effects of $\mathrm{Cr}$ supplementation on performance of dairy cows, but rather tried to answer the question whether $\mathrm{Cr}$ supplementation in the peripartal period will result in an metabolism-mediated increase in milk yield for the first 100 days of lactation as a standard performance parameter. A high variance of data and low per-group number of animals complicated the assessment of performance. Responsible for the lower mean performance in the experimental group was a lower milk yield in first-calvers resulting apparently from the selection of late-pregnant heifers for which no data on previous milk yield were available.

\section{Vliv dotace chromu na metabolismus dojnic v období kolem porodu}

Cílem pokusu bylo zjistit vliv dotace chromu (Cr) na metabolismus dojnic v období kolem porodu. $\mathrm{V}$ chovu Holštýnského skotu s průměrnou užitkovostí 7500 1, byl dojnicím podáván $\mathrm{Cr}$ ve formě kvasinek (Co-Factor III Chromium Yeast, Alltech, $0,1 \% \mathrm{Cr}^{3+}$ ) v dávce $10 \mathrm{mg} \mathrm{Cr/kus/den.} \mathrm{Aplikace} \mathrm{byla} \mathrm{zahájena} \mathrm{v} \mathrm{průměru} 21$ dní před porodem (a.p.) a ukončena 30 dní po porodu (p.p.). V průběhu pokusu byly odebírány vzorky krve a moči od dojnic pokusné $(\mathrm{E}, \mathrm{n}=10)$ a kontrolní skupiny $(\mathrm{C}, \mathrm{n}=10)$ v týdenních intervalech, byl sledován zdravotní stav pravidelným klinickým vyšetřením a užitkovost dojnic byla vyhodnocena za 100 dní laktace. Byl zjištěn pozitivní vliv podávání $\mathrm{Cr}$ na energetický metabolismus dojnic. U dojnic pokusné skupiny byla v poporodním období vyšší koncentrace glukózy, signifikantní rozdíly byly 4 týdny p.p. $(4,25 \pm 0,21$ vs. $3,74 \pm 0,36$ $\left.\mathrm{mmol} \cdot \mathrm{l}^{-1} ; p<0,01\right)$ a 5 týdnů p.p. $\left(4,06 \pm 0,41 \mathrm{vs.} 3,64 \pm 0,28 \mathrm{mmol} \cdot \mathrm{l}^{-1} ; p<0,05\right)$ a naopak nižší koncentrace ketolátek v krvi 4 týdny p.p. $\left(0,88 \pm 0,11\right.$ vs. $1,38 \pm 0,66 \mathrm{mmol} \cdot \mathrm{l}^{-1}$; $p<0,05)$. Dále byl zjištěn u skupiny dotované $\mathrm{Cr}$ nižší stupeň alterace jaterního parenchymu. U dojnic pokusné skupiny byla $\mathrm{v}$ poporodním období zjištěna nižší koncentrace bilirubinu 2 týdny p.p. $\left(3,93 \pm 0,84\right.$ vs. $\left.6,47 \pm 3,25 \mu \mathrm{mol} \cdot 1^{-1} ; p<0,05\right)$, nižší katalytická aktivita aspartátaminotransferázy 3 týdny p.p. $\left(1,37 \pm 0,14 \mathrm{vs.} 1,66 \pm 0,20 \mu \mathrm{kat} \cdot \mathrm{l}^{-1}\right.$; $p<0,01)$ a 5 týdnů po porodu $\left(1,16 \pm 0.08\right.$ vs. $\left.1,47 \pm 0,18 \mu \mathrm{kat} \cdot \mathrm{l}^{-1} ; p<0,01\right)$ a laktátdehydrogenázy 5 týdnů p.p. $\left(27,35 \pm 3,76\right.$ vs. $\left.33,61 \pm 5,61 \mu \mathrm{kat} \cdot{ }^{-1} ; p<0,05\right)$. Dotace $\mathrm{Cr}$ neovlivnila metabolismus dusíkatých látek, minerální metabolismus, koncentraci inzulinu v krevním séru. Koncentrace Cr v krvi nebyla dotací Cr ovlivněna. Po porodu došlo ke zvýšení vylučování Cr močí, vyšší hodnoty byly zjišš̌ny u dojnic dotovaných Cr 3 týdny p.p. $\left(7,14 \pm 1,72\right.$ vs. $\left.5,00 \pm 1,26 \mu \mathrm{g} \cdot \mathrm{l}^{-1} ; p<0,01\right)$ a 4 týdny p.p. $(8,40 \pm 3,13$ vs. $4,04 \pm 1,32$ $\left.\mu \mathrm{g} \cdot \mathrm{l}^{-1} ; p<0,01\right)$. I přes prokázaný pozitivní vliv Cr na energetický metabolismus $\mathrm{v}$ období kolem porodu nebyla ovlivněna užitkovost dojnic za prvních 100 dní laktace. Rovněž výskyt klinických onemocnění nebyl dotací Cr ovlivněn. 


\section{Acknowledgement}

This study was a part of the FRVŠ project No. 1226/99 and the project of the Ministry of Education, Youth and Sports of the Czech Republic No. 161700002.

\section{References}

ANDERSON, R. A. 1994: Stress efects on chromium nutrition of humans and farm animals. In: Biotechnology in the feed industry, Proceedings of Alltech's $10^{\text {th }}$ Annual Symposium, Lyons P., Jacques K. A. (eds). Nottingham University Press, pp.267-274

ANDERSON, R. A. 1995: Chromium, glucose tolerance, and lipid metabolism. J. Adv. Med. 8: 37

BESONG, S., JACKSON, J., TRAMMELL, S. AMARAL-PHILIPS, D. 1996: Effect of supplemental chromium picolinate on liver triglycerides, blood metabolites, milk yield, and milk composition in early-lactation cows. J. Dairy Sci. 79 (Suppl. 1): 97 (abstract)

BURTON, J. L., NONNECKE, B. J., ELSASSER, T. H., MALLARD, B. A., YANG, W. Z., MOWAT, D. N. 1995 Immunomodulatory activity of blood serum from chromium-supplemented periparturient dairy cows. Vet. Immunol. Immunopathol. 49: 29-38

CHANG, X., MALLARD, B. A., MOWAT, D. N. 1996: Effects of chromium on health status, blood neutrophil phagocytosis and in vitro lymphocyte blastogenesis of dairy cows. Vet. Immunol. Immunopathol. 52: 37-52

CHANG, X., MOWAT, D. N. 1992: Supplemental chromium for stressed feeder calves. J. Anim. Sci. 70: 559-565 DUCROS, V. 1992: Chromium metabolism. Biol. Trace Elem. Res. 32: 65-77

FRANK, A., DANIELSSON, R., JONES, B. 2000: Experimental copper and chromium deficiency and additional molybdenum supplementation in goats. II. Concentrations of trace and minor elements in liver, kidneys and ribs: haematology and clinical chemistry. Sci. Total Environ. 249: 143-170

HAYIRLI, A., BREMMER, D. R., BERTICS, S. J., SOCHA, M. T., GRUMMER, R. R. 2001: Effect of chromium supplementation on production and metabolic parameters in periparturient dairy cows. J. Dairy Sci. 84: 1218-1230

HRADECKY, P., JAGO_, P., JANÁK, J. 1978: Gas chromatografic head-space analysis of clinically interesting ketone bodies. J. Chrom. 146: 327 - 332

IRELAND, M. J. 1999: The effects of supplemental trivalent chromium of endocrine and immune system interactions of the periparturient dairy cow. MAI 37/04, p. 1158 (abstract)

KANEKO, J. J., HARVEY, J. W., BRUSS, M. L. et al. 1997: Clinical biochemistry of domestic animals. 5.ed., New York, Academic Press, 932 p.

LENTNER, C. 1981: Geigy Scientific Tables, Volume 1. CIBA-GEIGY Limited, Basle, 295 p.

MALLARD, B. A., BORGS, P. 1997: Effects of supplemental trivalent chromium on hormone and immune responses of cattle. In: Biotechnology in the feed industry, Proceedings of Alltech's $13^{\text {th }}$ Annual Symposium, Lyons P., Jacques K. A. (eds). Nottingham University Press, Nottingham, pp. 241-250

MERTZ, W. 1997: Confirmation: Chromium levels in serum, hair, and sweat decline with age. Nutr. Rev. 55: $373-$ 375

MOONSIE-SHAGER, S., MOWAT, D. N. 1993: Effects of level of supplemental chromium on performance, serum constituents, and immune status of stressed feeder calves. J. Anim. Sci. 71: 232-238

MORDENTI, A., PIVA, A., PIVA, G. 1997: The European perspective on organic chromium in animal nutrition. In: Biotechnology in the feed industry, Proceedings of Alltech's $13^{\text {th }}$ Annual Symposium, Lyons P., Jacques K. A. (eds). Nottingham University Press, pp. 227-240

MOWAT, D. N., CHANG, X., YANG, W. Z. 1993: Chelated chromium and bovine respiratory diseases vaccines for stressed feeder calves. Can. J. Anim. Sci. 73: 49-55

MOWAT, D. N., SUBIYATNO, A., YANG, W. Z. 1995: Chromium deficiency in first parity cows. In: Biotechnology in the feed industry, Proceedings of Alltech's $11^{\text {th }}$ Annual Symposium, Lyons P., Jacques K. A. (eds). Nottingham University Press, pp. 309-314

PAUSTENBACH, D. J., PANKO, J. M., FREDRICK, M. M., FINLEY, B. L., PROCTOR, D. M. 1997: Urinary chromium as a biological marker of environmental exposure: What are the limitations? Regul. Toxicol. Pharmacol. 26: 23-34

PECHOVÁ, A., ILLEK, J., HALOUZKA, R. 1997: Diagnosis and control of the development of hepatic steatosis in dairy cows in the periparturient period. Acta Vet. Brno 66: 235-243

SANO, H., MOWAT, D. N., BALL, R. O., TROUT, D. R. 1997: Effect of supplemental chromium on whole- body kinetics of glucose, lactate and propionate in rams fed a high grain diet. Comp. Biochem. Physiol. 118: 117-121

SAHIN, K., SAHIN, N., GULER, T., CERCI, I. H., ERKAL, N. 1996: Effect of chromium on animals grazing around the Elazig Ferrokrom Factory. Saglik-Bilimleri-Dergisi 10: 259-263 (abstract)

SUBIYATNO, A., MOWAT, D. N., LIPTRAP, R.. M. 1993: The effect of supplemental chromium on early lactation performance of Holstein cows. J. Dairy Sci. 76 (Suppl. 1): 304 (abstract)

SUBIYATNO, A., MOWAT, D. N., YANG, W. Z. 1996: Metabolite and hormonal responses to glucose or propionate infusions in periparturient dairy cows supplemented with chromium. J. Dairy Sci. 79: 1436-1445

UNDERWOOD E. J. 1977: Chromium. in Trace elements in human and animal nutrition. Fourth Edition, E. J. Underwood, ed. Academic Press, New York, pp. 258-270 
VILLALOBOS, J. A., ROMERO, C., TARRAGO, M. R., ROSADO, A. 1997: Supplementation with chromium picolinate reduces the incidence of placental retention in dairy cows. Can. J. Anim. Sci. 77: 329-330

VRZGULA et al. 1990: Poruchy látkového metabolismu hospodárskych zvierat a ich prevencia. Bratislava, Príroda, $2^{\text {nd }}$ edition, $503 \mathrm{p}$.

WILLIAMS, G. L. 1994: Using dietary fat supplementation to enhance reproductive potential in beef cows: fundamental and applied aspects. In: Infertility in Beef Cattle, Proc. Vet. Cont. Educ. Shortcourse, TX AM Univ., Coll. Vet. Med., pp. 56-84

YANG, W. Z., MOWAT, D. N., SUBIYATNO, A., LIPTRAP, R. M. 1996: Effects of chromium supplementation on early lactation performance of Holstein cows. Can. J. Anim. Sci. 76: 221-230 